\title{
Seismic retrofitting solution of an adobe masonry wall
}

\author{
Materials and Structures
}

January 2013, Volume 46, Issue 1-2, pp 203-219 | Cite as

- A. Figueiredo (1)

- H. Varum (1) Email author (hvarum@ua.pt)

- A. Costa (1)

- D. Silveira (1)

- C. Oliveira (1)

1. Departamento de Engenharia Civil, Universidade de Aveiro, Aveiro, Portugal

Original Article

First Online: 17_July 2012

Received: 22 March 2011

Accepted: 25 June 2012

- 952 Downloads

- 19_Citations

\begin{abstract}
Adobe constructions represent a high percentage of the national patrimony, with high historical, cultural and architectonic value. Well-preserved adobe structures can exhibit a particular architecture with very attractive geometric characteristics while also incorporating natural materials. However, the behavior of these structures is deficient under horizontal loads, such as those induced by an earthquake, which endangers their structural integrity and human lives. To develop a seismic retrofit solution, a real-scale wall was characterized and tested by considering permanent vertical actions with cyclic horizontal forces of increasing amplitude. To retrofit the wall, repair and seismic reinforcement solutions were developed and combined to evaluate their efficiencies. To repair the damages, hydraulic lime gum was injected by pressure into the cracks. The reinforcement solution included the use of a synthetic mesh in the wall. The retrofitted wall was then tested, and the results indicated that the retrofit solutions significantly improved the seismic performance of the wall. This study contributes to the characterization of walls constructed with adobe masonry and their behavior under horizontal actions. Furthermore, an economic, sustainable and efficient solution is presented for the retrofitting of adobe walls, with significant performance improvements obtained.
\end{abstract}

\section{Keywords}


Adobe structures Mechanical properties Structural behavior

Seismic assessment Seismic strengthening techniques

This is a preview of subscription content, $\underline{\log \underline{\text { in }} \text { to check access }}$

\section{Notes}

\section{Acknowledgments}

The authors of this paper would like to express their deepest thanks to all of the people, companies and institutions that allowed this research to be pursued by helping with the preparation and development of the experimental tests, including the Laboratory of Earthquake and Structural Engineering (LESE), Faculty of Engineering, Porto University; STAP; Fregaze; Aveiro City Council; and the Physics, Mechanical and Geological Sciences Departments of Aveiro University, all of which are located in Portugal.

\section{References}

1. Blondet M, Garcia G, Brzey S (2003) Construcciones de adobe resistentes a los terremotos. Contribution for "World-Housing Encyclopedia", Marjorie Greene, EERI/IAEE

Google Scholar (https://scholar.google.com/scholar?

q=Blondet\%20M\%2C\%20Garcia\%20G\%2C\%20Brzey\%20S\%20\%282003\%29

\%20Construcciones\%20de\%20adobe\%2oresistentes\%20a\%2olos\%2oterremot os.\%20Contribution\%20for\%20\%E2\%80\%9CWorld-

Housing\%2oEncyclopedia\%E2\%80\%9D\%2C\%20Marjorie\%20Greene\%2C\%20 EERI\%2FIAEE)

2. Blondet M, Torrealva D, Garcia G, Ginocchio F, Madueño I (2005) Using industrial materials for the construction of safe adobe houses in seismic areas. In: EarthBuild2005 International Conference, University of Technology.

Sydney, Australia

Google Scholar (https://scholar.google.com/scholar?

q=Blondet\%20M\%2C\%20Torrealva\%20D\%2C\%20Garcia\%20G\%2C\%20Ginocc hio\%20F\%2C\%20Madue\%C3\%B10\%20I\%20\%282005\%29\%2oUsing\%2oindus trial\%2omaterials\%2ofor\%2othe\%2oconstruction\%20of\%20safe\%20adobe\%2 ohouses\%20in\%20seismic\%20areas.\%20In\%3A\%20EarthBuild2005\%20Inter national\%20Conference\%2C\%2oUniversity\%20of\%2oTechnology.\%2oSydney $\% 2 \mathrm{C} \% 2 \mathrm{OAustralia)}$

3. Corazao M, Blondet M (1973) Estudio Experimental del Comportamiento Estructural de las Construcciones de Adobe Frente a Solicitaciones Sísmicas. Banco Peruano de los Constructores. Departamento de Ingenieria, Universidad Catolica del Peru, Lima, Perú

Google Scholar (https://scholar.google.com/scholar? q=Corazao\%20M\%2C\%2oBlondet\%20M\%20\%281973\%29\%20Estudio\%2oExp erimental\%2odel\%20Comportamiento\%2oEstructural\%2ode\%2olas\%20Const rucciones\%20de\%20Adobe\%2oFrente\%20a\%2oSolicitaciones\%20S\%C3\%ADs micas.\%20Banco\%20Peruano\%2ode\%2olos\%20Constructores.\%20Departame nto\%2ode\%2oIngenieria\%2C\%2oUniversidad\%2oCatolica\%2odel\%2oPeru\%2 C\%20Lima\%2C\%2oPer\%C3\%BA) 
4. Costa A, Varum H, Pereira H, Rodrigues H, Vicente R, Arêde A, Costa AA (2007) Avaliação experimental do comportamento fora do plano de paredes de alvenaria de adobe. V Seminário Arquitectura de Terra em Portugal, Departamento de Engenharia Civil, University of Aveiro, Aveiro, Portugal Google Scholar (https://scholar.google.com/scholar? q=Costa\%20A\%2C\%2oVarum\%20H\%2C\%2OPereira\%20H\%2C\%20Rodrigues \%20H\%2C\%2oVicente\%20R\%2C\%20Ar\%C3\%AAde\%20A\%2C\%20Costa\%2OA A\%20\%282007\%29\%20Avalia\%C3\%A7\%C3\%A30\%2oexperimental\%20do\%20 comportamento\%2ofora\%20do\%2oplano\%20de\%2oparedes\%20de\%20alvenar ia\%20de\%20adobe.\%20V\%20Semin\%C3\%A1rio\%20Arquitectura\%2ode\%20Te rra\%20em\%20Portugal\%2C\%2oDepartamento\%20de\%2oEngenharia\%20Civil \%2C\%2oUniversity\%20of\%20Aveiro\%2C\%20Aveiro\%2C\%2oPortugal)

5. Costa A, Varum H, Arêde A, Costa AA, Pereira H, Silveira D, Rodrigues H, Vicente R (2008) Caracterização do comportamento estrutural de adobes e de paredes de alvenaria de adobe. Portuguese Journal "Revista Construir", Workmedia, Ano V, Número 123, pp 22-25

Google Scholar (https://scholar.google.com/scholar? q=Costa\%20A\%2C\%20Varum\%2OH\%2C\%2OAr\%C3\%AAde\%2OA\%2C\%2OCost a\%20AA\%2C\%2oPereira\%20H\%2C\%20Silveira\%20D\%2C\%2oRodrigues\%20H \%2C\%2oVicente\%20R\%20\%282008\%29\%20Caracteriza\%C3\%A7\%C3\%A30\%2 odo\%20comportamento\%20estrutural\%20de\%20adobes\%20e\%20de\%2opare des\%2ode\%2Oalvenaria\%20de\%20adobe.\%2oPortuguese\%2OJournal\%20\%E2 \%80\%9CRevista\%20Construir\%E2\%80\%9D\%2C\%20Workmedia\%2C\%20Ano \%20V\%2C\%20N\%C3\%BAmero\%20123\%2C\%2opp\%2022\%E2\%80\%9325)

6. Fernandes M, Mestre V (2006) Portugal atlântico versus Portugal mediterrâneo - tipologias arquitectónicas em terra. International Conference TerraBrasil 2006, Ouro Preto, Minas Gerais

Google Scholar (https://scholar.google.com/scholar?

q=Fernandes\%20M\%2C\%2oMestre\%20V\%20\%282006\%29\%20Portugal\%20a tl\%C3\%A2ntico\%2oversus\%2oPortugal\%2omediterr\%C3\%A2neo\%20\%E2\%80 \%93\%2otipologias\%2oarquitect\%C3\%B3nicas\%20em\%20terra.\%2oInternatio nal\%20Conference\%2oTerraBrasil\%202006\%2C\%20Ouro\%2oPreto\%2C\%20 Minas\%20Gerais)

7. Figueiredo A (2009) Caracterização de uma solução de reforço sísmico de paredes de adobe. Civil Engineering Master Degree dissertation, Civil Engineering Department, Aveiro University, Aveiro Google Scholar (https://scholar.google.com/scholar? q=Figueiredo\%20A\%20\%282009\%29\%20Caracteriza\%C3\%A7\%C3\%A30\%2od e\%2ouma\%2osolu\%C3\%A7\%C3\%A30\%2ode\%2orefor\%C3\%A70\%20s\%C3\%AD smico\%2ode\%2oparedes\%2ode\%2oadobe.\%20Civil\%2oEngineering\%2oMaste r\%20Degree\%2odissertation\%2C\%20Civil\%2oEngineering\%2oDepartment\%2 C\%20Aveiro\%2oUniversity\%2C\%2oAveiro)

8. Grangeia C, Tareco H, Senos-Matias M, Varum H, Costa A (2010) Utilização de georadar na caracterização da evolução do dano estrutural de uma parede: ensaio à escala real. 8th National Conference on Seismology and Earthquake Engineering, Sísmica 2010, Universidade de Aveiro, Aveiro

Google Scholar (https://scholar.google.com/scholar? q=Grangeia\%20C\%2C\%2OTareco\%20H\%2C\%2OSenosMatias\%20M\%2C\%2oVarum\%20H\%2C\%2oCosta\%20A\%20\%282010\%29\%20 Utiliza\%C3\%A7\%C3\%A30\%2ode\%2ogeoradar\%2ona\%2ocaracteriza\%C3\%A7\% $\mathrm{C}_{3} \% \mathrm{~A} 30 \% 20 \mathrm{da} \% 20$ evolu\%C3\%A7\%C3\%A30\%20do\%2odano\%20estrutural\%2 ode\%20uma\%2oparede\%3A\%20ensaio\%20\%C3\%Ao\%20escala\%2oreal.\%208t 
h\%20National\%20Conference\%20on\%20Seismology\%20and\%2oEarthquake\% 20Engineering\%2C\%20S\%C3\%ADsmica\%202010\%2C\%2oUniversidade\%20de \%20Aveiro\%2C\%20Aveiro)

9. Houben H, Guillaud H (1994) Earth Construction-a Comprehensive guide, CRATerre - EAG. Intermediate Technology Publications, London

Google Scholar (http://scholar.google.com/scholar_lookup?

title=Earth\%20Construction\%E2\%80\%94a\%20Comprehensive\%2oguide\%2C \%20CRATerre\%20\%E2\%80\%93\%20EAG\&author=H.\%2OHouben\&author=H. \%20Guillaud\&publication_year=1994)

10. Kalagri A, Miltiadou-Fezans A, Vintzileou E (2010) Design and evaluation of hydraulic lime grouts for the strengthening of stone masonry historic structures. Mater Struct 43:1135-1146 CrossRef (https://doi.org/10.1617/s11527-009-9572-1) Google Scholar (http://scholar.google.com/scholar_lookup? title=Design\%20and\%20evaluation\%20of\%2ohydraulic\%2olime\%2ogrouts\%2 ofor\%20the\%20strengthening\%20of\%20stone\%20masonry\%20historic\%20str uctures\&author=A.\%20Kalagri\&author=A.\%20Miltiadou-

Fezans\&author $=$ E.\%20Vintzileou\&journal=Mater\%20Struct\&volume=43\&page S=1135-1146\&publication_year=2010)

11. Maheri M, Motielahi F, Najafgholipour MA (2011) The effects of pre and post construction moisture condition on the in-plane and out-of-plane strengths of brick walls. Mater Struct 44:541-559

CrossRef (https://doi.org/10.1617/s11527-010-9648-y) Google Scholar (http://scholar.google.com/scholar_lookup? title=The\%20effects\%20of\%2opre\%20and\%2opost\%20construction\%2omoist ure\%20condition\%20on\%20the\%2oin-plane\%20and\%20out-ofplane\%20strengths\%20of\%2obrick\%20walls\&author=M.\%20Maheri\&author= F.\%20Motielahi\&author=MA.\%2oNajafgholipour\&journal=Mater\%20Struct\&v olume $=44 \&$ pages $=541-559 \&$ publication_year $=2011$ )

12. Martins T, Varum H (2006) Adobe's mechanical characterization in ancient constructions: the case of Aveiro's region. Mater Sci Forum 514-516:1571-1575 CrossRef (https://doi.org/10.4028/www.scientific.net/MSF.514-516.1571) Google Scholar (http://scholar.google.com/scholar_lookup? title=Adobe\%E2\%80\%99s\%2omechanical\%20characterization\%2oin\%20ancie nt\%20constructions\%3A\%20the\%20case\%20of\%20Aveiro\%E2\%80\%99s\%20r egion\&author=T.\%20Martins\&author=H.\%20Varum\&journal=Mater\%20Sci\% 20 Forum\&volume $=514 \% \mathrm{E} 2 \% 80 \% 93516 \&$ pages $=1571-$ 1575\&publication_year=2006)

13. Minke G (2006) Building with earth, design and technology of a sustainable architecture. Birkhãuser-Publishers for Architecture, Basel-Berlin-Boston, ISBN-10: 3764374772

Google Scholar (https://scholar.google.com/scholar? q=Minke\%20G\%20\%282006\%29\%20Building\%20with\%2oearth\%2C\%2odesi gn\%20and\%2otechnology\%20of\%20a\%2osustainable\%20architecture.\%20Bir kh\%C3\%A3user\%E2\%80\%94Publishers\%2ofor\%20Architecture\%2C\%2oBaselBerlin-Boston\%2C\%20ISBN-10\%3A\%203764374772)

14. Mohammed A, Hughes TG (2011) Prototype and model masonry behavior under different loading conditions. Mater Struct 44:53-65 CrossRef (https://doi.org/10.1617/s11527-010-9608-6) Google Scholar (http://scholar.google.com/scholar_lookup? title=Prototype\%20and\%20model\%20masonry\%2obehavior\%2ounder\%2odiff 
erent\%2oloading\%20conditions\&author=A.\%20Mohammed\&author=TG.\%2O

Hughes\&journal $=$ Mater\%20Struct\&volume $=44$ \&pages $=53^{-}$

65\&publication_year=2011)

15. Noguez R, Navarro S (2005) Reparación de muros de adobe com el uso de mallas sintéticas. PUCP, International Conference SismoAdobe2005

Google Scholar (https://scholar.google.com/scholar?

q=Noguez\%20R\%2C\%20Navarro\%20S\%20\%282005\%29\%20Reparaci\%C3\%B 3n\%2ode\%2omuros\%2ode\%20adobe\%20com\%2oel\%2ouso\%2ode\%2omallas \%20sint\%C3\%A9ticas.\%20PUCP\%2C\%2OInternational\%2oConference\%20Sis moAdobe2005)

16. Pereira H (2008) Caracterização do comportamento estrutural de construções em adobe. Civil Engineering Master Degree dissertation, Civil Engineering Department, Aveiro University, Aveiro

Google Scholar (https://scholar.google.com/scholar?

q=Pereira\%20H\%20\%282008\%29\%20Caracteriza\%C3\%A7\%C3\%A30\%2odo\% 2ocomportamento\%2oestrutural\%20de\%2oconstru\%C3\%A7\%C3\%B5es\%2oem \%20adobe.\%20Civil\%2oEngineering\%2oMaster\%2oDegree\%2odissertation\%2 C\%20Civil\%2oEngineering\%2oDepartment\%2C\%20Aveiro\%2oUniversity\%2C \%20Aveiro)

17. Silveira D, Varum H, Costa A (2007) Rehabilitation of an important cultural and architectural heritage: the traditional adobe constructions in Aveiro district. In: Kungolas A, Brebbia CA, Beriatos E (eds) Sustainable development. WITPress, Carvoeiro, Algarve, pp 705-714. ISBN 978-1-84564-103-0

Google Scholar (https://scholar.google.com/scholar?

q=Silveira\%20D\%2C\%20Varum\%20H\%2C\%20Costa\%20A\%20\%282007\%29\% 2oRehabilitation\%20of\%20an\%2oimportant\%20cultural\%20and\%2oarchitect ural\%2oheritage\%3A\%20the\%2otraditional\%20adobe\%2oconstructions\%2oin \%20Aveiro\%2odistrict.\%2OIn\%3A\%2oKungolas\%20A\%2C\%2oBrebbia\%2oCA \%2C\%20Beriatos\%20E\%20\%28eds\%29\%20Sustainable\%2odevelopment.\%20 WITPress\%2C\%20Carvoeiro\%2C\%2OAlgarve\%2C\%20pp\%20705\%E2\%80\%93 714.\%20ISBN\%20978-1-84564-103-0)

18. Silveira D, Varum H, Costa A, Martins T, Pereira H, Almeida J (2012) Mechanical properties of adobe bricks in ancient constructions. Constr Build Mater 28(1):36-44. doi: 10.1016/j.conbuildmat.2011.08.046 (https://doi.org/10.1016/j.conbuildmat.2011.08.046) CrossRef (https://doi.org/10.1016/j.conbuildmat.2011.08.046) Google Scholar (http://scholar.google.com/scholar_lookup? title=Mechanical\%2oproperties\%20of\%20adobe\%2obricks\%2oin\%20ancient\% 20constructions\&author=D.\%20Silveira\&author=H.\%2oVarum\&author=A.\%2 oCosta\&author=T.\%20Martins\&author=H.\%20Pereira\&author=J.\%20Almeida \&journal $=$ Constr\%2oBuild\%20Mater\&volume $=28 \&$ issue $=1 \&$ pages $=36-$ 44\&publication_year $=2012 \&$ doi $=10.1016 \% 2 F j$.conbuildmat.2011.08.046)

19. Tareco H, Grangeia C, Varum H, Senos-Matias M (2009) A high resolution GPR experiment to characterize the internal structure of a damaged adobe wall. EAGE First Break 27(8):79-84

Google Scholar (http://scholar.google.com/scholar_lookup? title=A\%2ohigh\%2oresolution\%20GPR\%20experiment\%20to\%2ocharacterize \%2othe\%2ointernal\%20structure\%20of\%20a\%2odamaged\%20adobe\%20wall \&author=H.\%20Tareco\&author=C.\%20Grangeia\&author=H.\%20Varum\&auth or=M.\%20Senos-

Matias\&journal=EAGE\%2oFirst\%20Break\&volume=27\&issue=8\&pages=7984\&publication_year=2009) 
20. Torgal P, Jalali S (2009) Construção em Terra: o Passado, Presente e o Futuro Revista Profissional da construção. National Journal Arte \&Construção, N. 230 - Portugal

Google Scholar (https://scholar.google.com/scholar?

q=Torgal\%20P\%2C\%20Jalali\%20S\%20\%282009\%29\%20Constru\%C3\%A7\%C

3\%А30\%20em\%2oTerra\%3A\%200\%2oPassado\%2C\%2oPresente\%20e\%200\%2 oFuturo\%20\%E2\%80\%93\%20Revista\%2oProfissional\%20da\%2oconstru\%C3

\%A7\%C3\%A30.\%20National\%20Journal\%20Arte\%20\%26Constru\%C3\%A7\%C 3\%A30\%2C\%20N.\%20230\%20\%E2\%80\%93\%20Portugal)

21. Vargas J, Blondet M, Ginocchio F, García G (2005) 35 Años de investigaciones en sismo adobe: la tierra armada, International Conference SismoAdobe 2005: Architecture, Construction and Conservation of Earthen Buildings in Seismic Areas, Lima, Pontificia Universidad Católica del Perú,. Lima, Peru

Google Scholar (https://scholar.google.com/scholar? q=Vargas\%20J\%2C\%2oBlondet\%20M\%2C\%20Ginocchio\%2oF\%2C\%20Garc\% C3\%ADa\%20G\%20\%282005\%29\%2035\%20A\%C3\%B1os\%20de\%2oinvestigaci ones\%20en\%20sismo\%20adobe\%3A\%2ola\%2otierra\%2oarmada\%2C\%2oInter national\%20Conference\%20SismoAdobe\%202005\%3A\%20Architecture\%2C\% 20Construction\%20and\%20Conservation\%20of\%2oEarthen\%20Buildings\%20 in\%20Seismic\%2OAreas\%2C\%2oLima\%2C\%2oPontificia\%2oUniversidad\%20 Cat\%C3\%B3lica\%2odel\%2oPer\%C3\%BA\%2C.\%2oLima\%2C\%2oPeru)

22. Varum H, Martins T, Velosa A (2005a) Caracterização do adobe em construções existentes na região de Aveiro. Iberian-American Conference, IV SIACOT Seminário Ibero-Americano de Construção com terra e III Seminário Arquitectura de Terra em Portugal. Convento da Orada. Monsaraz, Portugal Google Scholar (https://scholar.google.com/scholar?

q=Varum\%20H\%2C\%2oMartins\%20T\%2C\%2oVelosa\%20A\%20\%282005a\%2 9\%20Caracteriza\%C3\%A7\% 3 \%A30\%20do\%20adobe\%20em\%2oconstru\%C3 \%A7\%C3\%B5es\%20existentes\%20na\%20regi\%C3\%A30\%20de\%20Aveiro.\%2OI berian-

American\%2oConference\%2C\%2oIV\%20SIACOT\%20Semin\%C3\%A1rio\%20Ib ero-

Americano\%2ode\%20Constru\%C3\%A7\%C3\%A30\%20com\%20terra\%20e\%20II I\%20Semin\%C3\%A1rio\%20Arquitectura\%20de\%20Terra\%20em\%20Portugal. \%20Convento\%20da\%20Orada.\%20Monsaraz\%2C\%20Portugal)

23. Varum H, Costa A, Pereira H, Almeida J, Rodrigues H (2006a) Avaliação Experimental do Comportamento Estrutural de Elementos Resistentes em Alvenaria de Adobe - III Congreso Internacional de Arquitectura en Tierra: Tradición y Innovacón - Universidad de Valladolid, Valladolid, España, 11 a 12 de Agosto de 2006

Google Scholar (https://scholar.google.com/scholar? q=Varum\%20H\%2C\%2oCosta\%2oA\%2C\%2oPereira\%2oH\%2C\%2oAlmeida\%2 oJ\%2C\%2oRodrigues\%20H\%20\%282006a\%29\%20Avalia\%C3\%A7\%C3\%A3o \%2oExperimental\%20do\%2oComportamento\%2oEstrutural\%20de\%2oEleme ntos\%2oResistentes\%20em\%2oAlvenaria\%2ode\%2oAdobe\%20\%20III\%20Congreso\%2oInternacional\%20de\%20Arquitectura\%20en\%2oTierr a\%3A\%2oTradici\%C3\%B3n\%20y\%2oInnovac\%C3\%B3n\%20\%20Universidad\%2Ode\%2oValladolid\%2C\%2oValladolid\%2C\%2oEspa\%C3\%B 1a\%2C\%2011\%20a\%2012\%2ode\%2oAgosto\%20de\%202006)

24. Varum H, Costa A, Pereira H, Almeida J (2006b) Comportamento estrutural de elementos resistentes em alvenaria de adobe. International ConferenceTerra Brasil. Minas Gerais. Ouro Preto, Brazil 
Google Scholar (https://scholar.google.com/scholar?

q=Varum\%20H\%2C\%20Costa\%20A\%2C\%2oPereira\%20H\%2C\%2OAlmeida\%2 oJ\%20\%282006b\%29\%20Comportamento\%2oestrutural\%2ode\%20elementos \%2oresistentes\%20em\%20alvenaria\%20de\%20adobe.\%2oInternational\%20Co nferenceTerra\%2oBrasil.\%20Minas\%20Gerais.\%20Ouro\%20Preto\%2C\%2oBra zil)

25. Varum H, Costa A, Velosa A, Martins T, Pereira H, Almeida J (2006c) "Caracterização mecânica e patológica das construções em Adobe no distrito de Aveiro como suporte em intervenções de reabilitação" - Projecto Culture 2000/Mediterrae - Houses and cities built with earth: conservation, significance and urban quality. ARGUMENTUM, Junho de 2006, pp 41-45, ISBN 972-8479-41-7

Google Scholar (https://scholar.google.com/scholar? q=Varum\%20H\%2C\%20Costa\%20A\%2C\%2OVelosa\%2oA\%2C\%2oMartins\%20 T\%2C\%2oPereira\%20H\%2C\%2oAlmeida\%20J\%20\%282006c\%29\%20\%E2\%8 о\%9CCaracteriza\%C3\%A7\%C3\%A30\%2omec\%C3\%A2nica\%2oe\%2opatol\%C3 \%B3gica\%2odas\%20constru\%C3\%A7\%C3\%B5es\%20em\%20Adobe\%20no\%20 distrito\%2ode\%20Aveiro\%20como\%2osuporte\%20em\%2ointerven\%C3\%A7\% C3\%B5es\%2ode\%2oreabilita\%C3\%A7\%C3\%A30\%E2\%80\%9D\%2O-

\%20Projecto\%20Culture\%202000\%2FMediterrae\%20-

\%20Houses\%20and\%20cities\%2obuilt\%20with\%20earth\%3A\%20conservation \%2C\%20significance\%20and\%2ourban\%20quality.\%20ARGUMENTUM\%2C\% 20Junho\%2Ode\%2O2006\%2C\%2Opp\%2041\%E2\%80\%9345\%2C\%20ISBN\%20 972-8479-41-7)

26. Varum H, Figueiredo A, Silveira D, Martins T, Costa A (2011) Outputs from the research developed at the University of Aveiro regarding the mechanical characterization of existing adobe constructions in Portugal - Revista Informes de la Construcción 63(523):127-142. doi: $\underline{\text { 10.3989/ic.10.016 }}$

(https://doi.org/10.3989/ic.10.016)

27. Veiga O, Galhano F (1992) Arquitectura Tradicional Portuguesa. Portugal de Perto Publicações D. Quixote, Portugal Google Scholar (http://scholar.google.com/scholar_lookup? title=Arquitectura\%20Tradicional\%20Portuguesa\&author=0.\%20Veiga\&autho r=F.\%20Galhano\&publication_year=1992)

28. Yamin L, Phillips C, Reyes J, Rivero S, Ruiz D (2007) Estudios de vulnerabilidad sísmica, rehabilitación y refuerzo de casas en adobe y tapia pisada. Revista de Estudios sobre Património. J Cult Heritage Stud, ISSN 16579763

Google Scholar (https://scholar.google.com/scholar?

q=Yamin\%20L\%2C\%2oPhillips\%20C\%2C\%2oReyes\%20J\%2C\%2oRivero\%20S \%2C\%20Ruiz\%20D\%20\%282007\%29\%20Estudios\%20de\%20vulnerabilidad\% 20s\%C3\%ADsmica\%2C\%2orehabilitaci\%C3\%B3n\%2oy\%2orefuerzo\%2ode\%2o casas\%20en\%20adobe\%20y\%2otapia\%2opisada.\%2oRevista\%2ode\%2oEstudi os\%20sobre\%2oPatrim\%C3\%B3nio.\%20J\%20Cult\%2oHeritage\%2oStud\%2C\% 20ISSN\%201657-9763)

29. Zavala C, Igarashi L (2005) Propuesta de Reforzamiento para Muros de Adobe. In: International Conference SismoAdobe 2005: architecture, construction and conservation of earthen buildings in seismic areas. Lima, Pontificia Universidad Católica del Perú, Lima, Peru

Google Scholar (https://scholar.google.com/scholar? q=Zavala\%20C\%2C\%2OIgarashi\%20L\%20\%282005\%29\%20Propuesta\%20de \%20Reforzamiento\%2opara\%20Muros\%20de\%20Adobe.\%20In\%3A\%2oInter 
national\%20Conference\%20SismoAdobe\%202005\%3A\%20architecture\%2C\%2 oconstruction\%20and\%20conservation\%20of\%20earthen\%2obuildings\%2oin \%20seismic\%20areas.\%20Lima\%2C\%20Pontificia\%2oUniversidad\%20Cat\%C3 \%B3lica\%20del\%2oPer\%C3\%BA\%2C\%2OLima\%2C\%2OPeru)

\section{Copyright information}

(C) RILEM 2012

\section{About this article}

Cite this article as:

Figueiredo, A., Varum, H., Costa, A. et al. Mater Struct (2013) 46: 203. https://doi.org/10.1617/s11527-0129895-1

- DOI (Digital Object Identifier) https://doi.org/10.1617/s11527-012-9895-1

- Publisher Name Springer Netherlands

- Print ISSN 1359-5997

- Online ISSN 1871-6873

- About this journal

- Reprints and Permissions

페케이

Published in cooperation with

the International Union of Laboratories and Experts in Construction Materials Systems and Structures

\section{Personalised recommendations}


1. Seismic behavior of two Portuguese adobe buildings: Part I - in-plane cyclic testing of a full-scale adobe wall

Silveira, Dora... Monteiro, Ricardo

International Journal of Architectural Heritage (2018)

2. Seismic performance of traditional adobe masonry walls subjected to in-plane cyclic loading

Wu, Feng... Li, Hong-Nan

Materials and Structures (2017)

3. Seismic Vulnerability Mitigation of a Masonry Church by Means of CFRP Retrofitting

Milani, Gabriele... Valente, Marco

Procedia Engineering (2017)

Want recommendations via email? Sign up now Powered by: Recommended $\mathbf{R}$

\section{SPRINGER NATURE}

(C) 2017 Springer International Publishing AG. Part of $\underline{\text { Springer Nature. }}$

Not logged in Not affiliated 95.92.96.228 